\title{
Continuous Vagus Nerve Monitoring during Carotid Endarterectomy
}

\author{
Tamaki Tomonori1 ${ }^{*}$, Kubota Minoru², Node Yoji', Morita Akio ${ }^{3}$ \\ ${ }^{1}$ Departmrnt of Neurological Surgery, Nippon Medical School, Tamanagayama Hospital, Tokyo, Japan \\ ${ }^{2}$ Department of Clinical Laboratory, Nippon Medical School, Tamanagayama Hospital, Tokyo, Japan \\ ${ }^{3}$ Departmrnt of Neurological Surgery, Nippon Medical School, Tokyo, Japan \\ Email: *tamakito@nms.ac.jp
}

How to cite this paper: Tomonori, T., Minoru, K., Yoji, N. and Akio, M. (2017) Continuous Vagus Nerve Monitoring during Carotid Endarterectomy. Open Journal of Modern Neurosurgery, 7, 1-9. http://dx.doi.org/10.4236/ojmn.2017.71001

Received: August 7, 2016

Accepted: December 9, 2016

Published: December 12, 2016

Copyright (C) 2017 by authors and Scientific Research Publishing Inc. This work is licensed under the Creative Commons Attribution International License (CC BY 4.0).

http://creativecommons.org/licenses/by/4.0/ (c) (i) Open Access

\begin{abstract}
Backgrounds: Injury to the vagus nerve or one of its branches during carotid endarterectomy can result in vocal fold paralysis but the exact mechanism of injury responsible for vocal fold paralysis after carotid endarterectomy is unclear. Aims: This study was performed to identify potential predictors of vagus nerve injury and obtain feedback by application of intraoperative continuous vagus nerve monitoring. Materials and Methods: Seventy-four patients undergoing carotid endarterectomy were enrolled. A new vagus nerve electrode was designed for less invasive continuous vagus nerve stimulation and monitoring of the vocal fold electromyogram without disturbing the surgical procedure. The device was rectangular $(13 \mathrm{~mm} \times 9 \mathrm{~mm})$, with two small round electrodes set on a flexible silicon plate and tube. The electrode was fully implantable during carotid endarterectomy and was positioned at the most distal site of the vagus nerve by suturing to the connective tissue without nerve dissection. All patients underwent laryngoscopy to assess postoperative vocal fold and pharyngeal wall palsy at one week after carotid endarterectomy. Results: Sudden loss of the vocal fold electromyogram was noted in two patients (during plaque removal and during arterial wall suture in one each). In these two patients, incomplete vocal fold and pharyngeal palsy was confirmed by laryngoscopy. The cause of vagus nerve injury may have been traction at the time of distal internal carotid artery manipulation. The vocal fold electromyogram remained normal during the operation in the other 72 patients. However laryngoscopy revealed postoperative vocal fold and pharyngeal palsy in six patients. These findings suggested that delayed vagus nerve injury can occur after carotid endarterectomy. Conclusion: The continuous vagus nerve monitoring may be worthwhile for elucidating the mechanism of vagus nerve injury related to carotid endarterectomy.
\end{abstract}

\section{Keywords}

Carotid Endarterectomy, Vagus Nerve, Vocal Fold, Neuromonitoring, Complication 


\section{Introduction}

Injury to the vagus nerve ( $\mathrm{VN})$ is a well-recognized problem in patients undergoing carotid endarterectomy (CEA) [1] [2]. VN damage can lead to undesirable consequences, including vocal disturbance, aspiration, and dysphagia. Although VN palsy is a serious complication of CEA, the causes of VN injury are not well understood [3] [4]. In patients undergoing neck surgery, intraoperative neuromonitoring has been advocated as a method of localizing and identifying the recurrent laryngeal nerve (RLN), as well as being used to predict postoperative vocal fold function [5] [6]. We previously reported a method of intermittent VN monitoring during CEA using a hand-held monopolar electrode for VN stimulation [7]. However, the hand-held stimulator had certain limitations. Besides difficulty in precisely identifying the $\mathrm{VN}$, assessment of functional integrity was limited to the periods of intermittent stimulation. To overcome these limitations, a method that allowed continuous monitoring would be desirable. Accordingly, we performed the present investigation to assess the effectiveness of a new flexible plate electrode for continuous VN monitoring (VNM) by retrospective evaluation in 74 patients undergoing CEA.

\section{Materials and Methods}

Patients were informed that the continuous VNM system would be employed to assist in localization and identification of the $\mathrm{VN}$, as well as to assess $\mathrm{VN}$ function during surgery, and written informed consent was obtained from each patient. There are no financial or professional associations between the authors and the manufacturer of the VNM system. From April 2012 to June 2015, we performed CEA in 96 patients. In 3 patients, continuous VNM could not be done due to technical problems. In addition, we could not identify the VN in 18 patients and 1 patient needed re-exploration for wound hematoma. We excluded these 22 patients. The remaining 74 patients successfully underwent continuous VNM, including 69 men and 5 women aged from 64 to 88 years (mean age: 76 years). Among them, 39 patients underwent right CEA and 35 had left CEA. Induction of anesthesia was achieved with remifentanyl $(2 \mathrm{mg} / \mathrm{kg})$ and propofol (2 - $4 \mathrm{mg} / \mathrm{kg}$ ), and neuromuscular blockade was established. Then a Xomed Nerve Integrity Monitor Electromyogram (EMG) endotracheal tube (Medtronic, Jacksonville, Fila) was inserted by the anesthesiologist. The tube was placed by direct laryngoscopy so that the center of the blue region (indicating the electrodes) was in contact with the true vocal fords and then was it fixed at the angle of the mouth on the non-operated side. Rotation of the tube could be detected by changes of EMG impedance and was corrected by the anesthesiologist. No additional muscle relaxant was given after the first dose. The cuff pressure was maintained at $20-22 \mathrm{~cm} \mathrm{H}_{2} \mathrm{O}$. After moving the head and neck from the neutral position to full extension, we checked monitor function and the location of the endotracheal tube electrodes by confirming that (1) the impedance was $<25 \mathrm{k} \Omega$ with an impedance imbalance $<5.0 \mathrm{k} \Omega$ and (2) there was a normal baseline. The neck was maintained in moderate extension until the end of the operation. In all 
patients, CEA was performed by the same standard surgical technique, which involved dissection of the carotid artery while causing the minimum possible damage to any cranial nerves in the operating field. Special attention was paid to careful dissection of the VN from the carotid artery, especially at the sites of proximal and distal clamping. We did not use self-retaining wound retractors. An internal shunt and a Hemashield patch graft were employed in all patients, and the procedure was always performed under an operating microscope. Our intention was to develop a less invasive flexible plate VN stimulation electrode that would not disturb the CEA procedure. The electrode was designed so that dissection of the $\mathrm{VN}$ was not required for attachment and so that it remained in contact with the nerve for continuous VNM. Accordingly, two small round stimulation electrodes (cerebral surface electrodes for motor evoked potential monitoring during craniotomy) were placed on a flexible silicon plate and tube (Figure 1). The electrode was fully implantable during CEA and was connected to a Nihon Kohden MEB-2312. Prior to positioning this continuous VNM electrode, we used a conventional handheld bipolar stimulation electrode (stimulus intensity of $0.5-2.0 \mathrm{~mA}$, pulse width of $100 \mu \mathrm{s}$, and frequency of $3 \mathrm{~Hz}$ ) to identify the $\mathrm{VN}$ and we placed the continuous VNM electrode at the most caudal point of the nerve. To maintain contact with the $\mathrm{VN}$, we sutured the silicon plate of the electrode to connective tissue (Figure 2). The stimulus conditions for continuous VNM were as follows: sense: $500 \mu \mathrm{V}$, filter: $2 \mathrm{KHz} \sim 50$ $\mathrm{Hz}$, stimulus intensity: $<2 \mathrm{~mA}$, single train, duration: $0.2 \mathrm{msec}$, stimulation rate: $1 \mathrm{~Hz}$, number of stimuli: 10, monitoring time (/div): $20 \mathrm{msec}$, analysis time (/div): $3 \mathrm{msec}$, and tend interval: $1 \sim 3$ minutes. The initial stimulus intensity was $0.5 \mathrm{~mA}$ using a negative square wave impulse and it was gradually increased in $0.5-\mathrm{mA}$ intervals to the upper limit of $2 \mathrm{~mA}$ until the first stable EMG signal from the vocal fold was identified to evaluate the influence of signal amplitude.
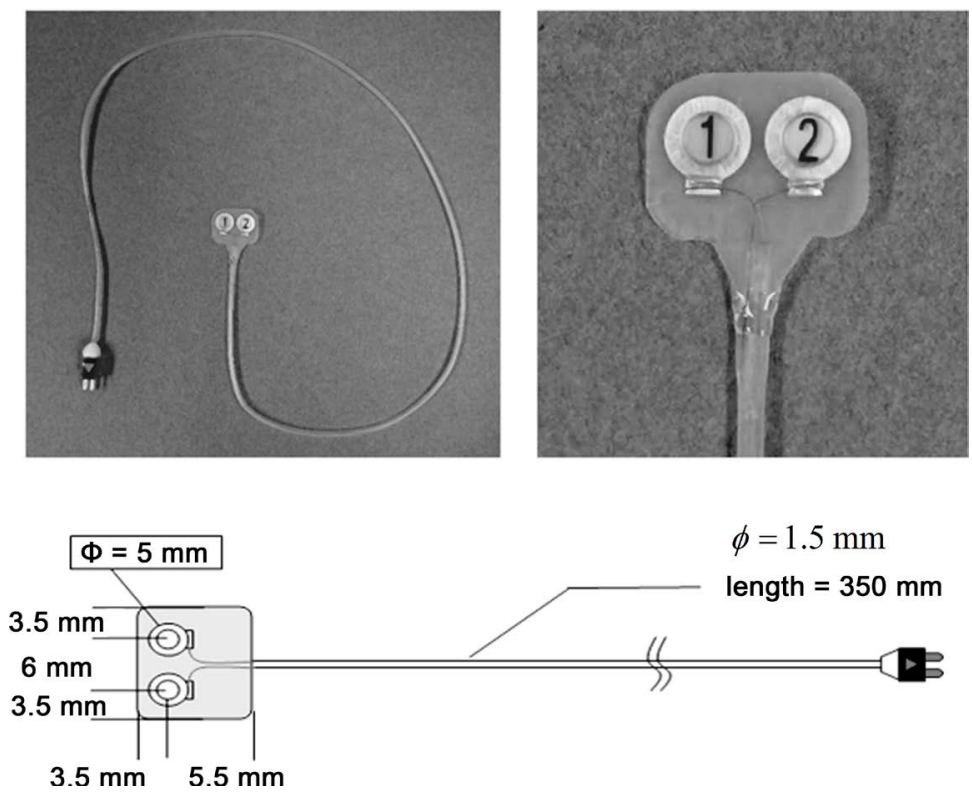

Figure 1. This photograph shows the new stimulation electrode for continuous vagus nerve monitoring. 


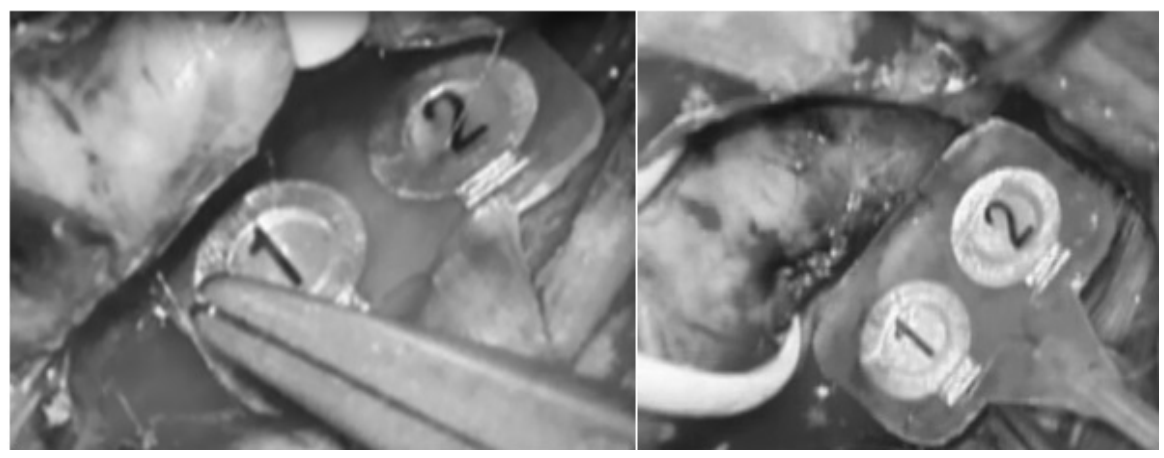

Figure 2. This photograph shows using the new stimulation electrode during carotid endarterectomy.

The Nihon Kohden MEB-2312 was used for both stimulation and measurement, with the latency, amplitude, and duration being assessed as quantitative parameters. Latency was defined as the time (in milliseconds) between the stimulus artifact and the onset of EMG activity and amplitude was defined as the magnitude of the EMG wave (in microvolts). Data are expressed as the mean \pm standard deviation. EMG changes were classified as signal loss (a signal was initially obtained from the $\mathrm{VN}$, but could not be elicited subsequently at $2.5 \mathrm{~mA}$ and an event threshold of $100 \mathrm{mV}$ ) or impairment (latency delayed by less than $20 \%$ and amplitude decreased by less than 50\%). For evaluation of cardiac and pulmonary side effects, the heart rate, blood pressure, and $\mathrm{SaO}_{2}$ were measured before, during, and after continuous VNM. The electrode was removed from the VN just before wound closure. In all patients, vocal fold and pharyngeal movements were observed by laryngoscopy at one week after CEA. When vocal fold dysfunction was identified, follow-up was done at 2-month intervals. We used the Student's t-test for continuous variables and P-values $<0.05$ were considered statistically significant.

\section{Results}

The outcome of surgery was favorable in all patients and none of them developed cerebral ischemia. The mean operating times was $164 \mathrm{~min}$. Heart rate, blood pressure, and $\mathrm{SaO}_{2}$ did not show significant changes during continuous VNM. Intraoperative electrode dislocation did not occur. The mean continuous VNM time was $68 \mathrm{~min}$ (range: 48 - $108 \mathrm{~min}$ ). A stable initial EMG response could be elicited from $74 \mathrm{VNs}$ during CEA, with a mean supramaximal current of $1.3 \mathrm{~mA}$ (range: $0.5-2.0 \mathrm{~mA}$ ). Evoked potentials were successfully recorded from the vocal fold muscle via surface electrodes. Mean values for the left VN obtained by continuous VNM were an amplitude of $474 \pm 142 \mu \mathrm{V}$, latency of $10.3 \pm 1.8 \mathrm{~ms}$, and duration of $8.7 \pm 2.3 \mathrm{~ms}$, while the mean values for the right $\mathrm{VN}$ were an amplitude of $486 \pm 323 \mu \mathrm{V}$, latency of $7.5 \pm 0.8 \mathrm{~ms}$, and duration of $8.4 \pm 2.7 \mathrm{~ms}$. The mean latency of the right $\mathrm{VN}$ was significantly shorter $(\mathrm{p}<$ 0.001 ) than that of the left $\mathrm{VN}$, whereas the duration and amplitude were similar for both VNs. EMG signal loss occurred in two patients, being noted during plaque removal and during suture of the distal internal carotid artery (ICA) in 
one case each (Figure 3). In these two patients, we could not detect the EMG by stimulating the VN at any site after signal loss. Laryngoscopy demonstrated incomplete vocal fold and pharyngeal wall palsy, with recovery after 6 months in one patient and 12 months in the other. In the remaining 72 CEA procedures, there was no signal loss or impairment of the vocal fold EMG during continuous VNM. Although six patients showed incomplete vocal fold and pharyngeal palsy on laryngoscopy, they recovered after 2 to 12 months (Figure 4). There were no complications of continuous VNM.

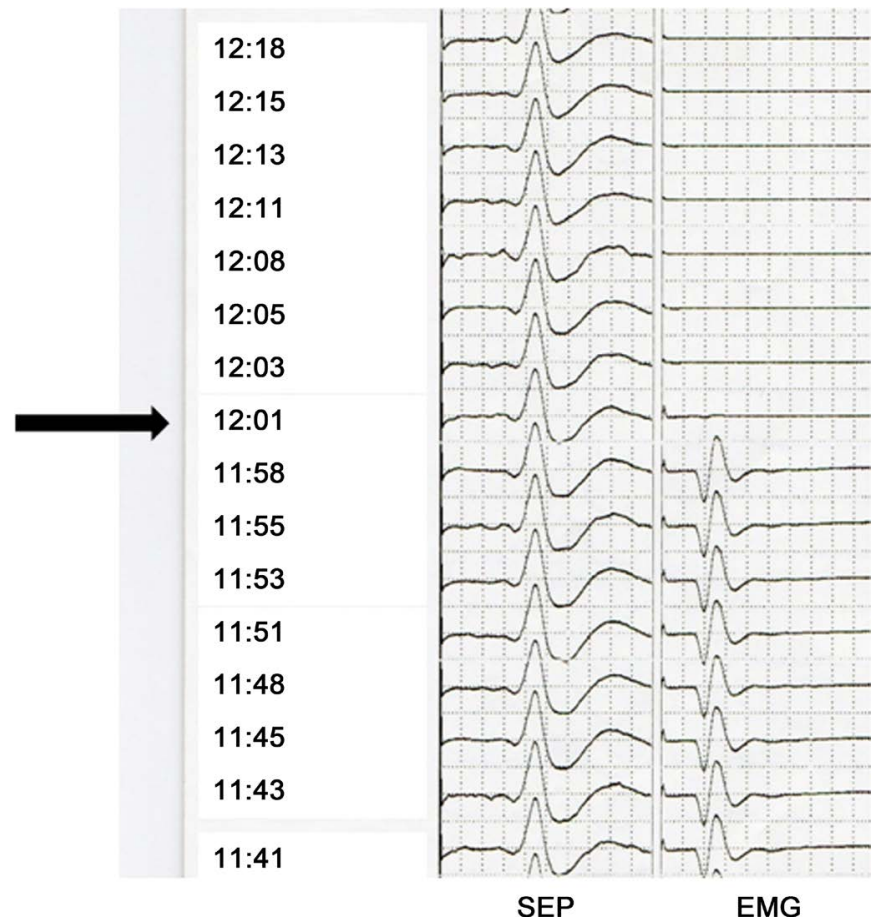

Figure 3. The sensory evoked potential (left side) and ipsilateral vocal fold electromyogram (right side) were revealed. The black arrow shows vocal fold electromyogram disappeared suddenly during plaque removing. SEP: Sensory evoked potential, EMG: Vocal fold electromyogram.

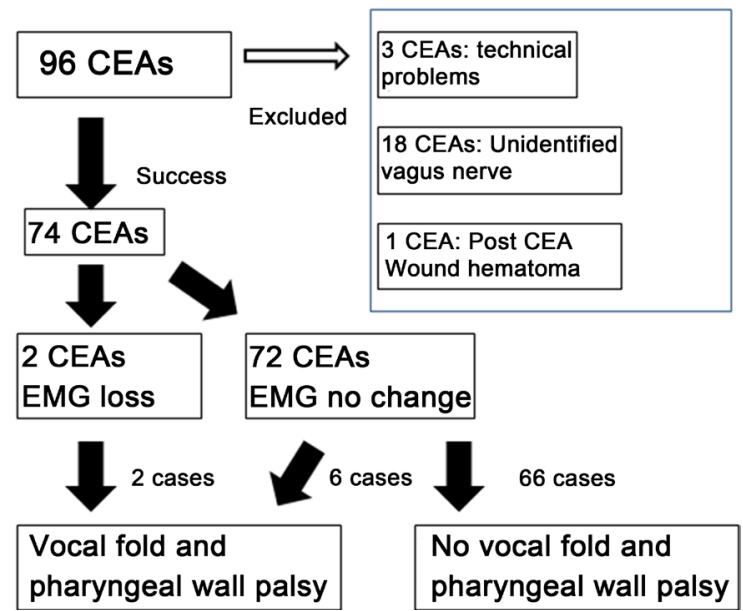

Figure 4. This figure shows the sumary of vocal fold electromyogram and laryngoscope findings. 


\section{Discussion}

The reported causes of VN injury during CEA include transection, clamping, stretching, electro-thermal injury, ligature entrapment, and ischemia, but the actual cause of VN injury is often unclear and unproven [1] [2] [8] [9]. Our continuous VNM method is one approach for providing data on the mechanism of VN injury during CEA. In our series, sudden loss of the EMG occurred in two patients during plaque removal and arterial suture, respectively, but not during connective tissue dissection before confirming the VN. The site of VN injury after CEA is important. We previously reported that CEA was associated with injury to the VN trunk in the parapharyngeal space based on data obtained by magnifying laryngoscopy [9]. In the present study, VN injury also affected the nerve trunk in the parapharyngeal space since laryngoscopy revealed vocal fold and pharyngeal wall palsy. A possible mechanism of VN injury during CEA is as follows. The VN trunk in the parapharyngeal space is located near the upper border of the operative field for CEA, just posterior to the internal carotid artery (ICA) [8]. Therefore, ICA manipulation (plaque removal and distal arterial suture in our patients) could stretch the $\mathrm{VN}$ together with the vessel and cause traction injury. Six of our patients developed postoperative VN palsy, although the vocal fold EMG was normal at the completion of CEA and impairment of the EMG signal (increasing latency and decreasing amplitude) did not occur intraoperatively. This suggests that VN injury does not only occur during surgery, and that delayed nerve injury is possible after CEA. Such delayed injury to the VN after wound closure could be related to compression by local hematoma or connective tissue edema, or to $\mathrm{VN}$ trunk ischemia, and may occur from a few hours to several days after CEA. We previously reported the detection of three cases of Vernet's syndrome by magnifying laryngoscopy after CEA. The glossopharyngeal nerve and accessory nerve are not confirmed during CEA and may sometimes be injured [8]. It is possible that manipulation of the distal portion of the ICA may indirectly damage any of these three nerves (VN, glossopharyngeal nerve, and accessory nerve) by causing edema, swelling, and hemorrhage in the parapharyngeal space. Before this study, we hypothesized that intraoperative impairment of the EMG could provide effective feedback on the effects of surgical maneuvers and predict VN function after CEA, but we did not find such immediate EMG changes and the present monitoring method was not so useful for protecting the VN. There were several limitations of our continuous VNM method. The first limitation was that we could not place the electrode distal to the predicted site of injury to the VN because we usually could not see the nerve in the parapharyngeal space during CEA. Therefore, we could not monitor VN function distal to the predicted site of injury. Second, we could not place the electrode before identifying the VN. Although there were no cases of EMG impairment or failure to obtain a signal at the start of continuous VNM in this series, if the VN was injured before confirmation we would not be able to document the injury. Third, we could not identify the VN in all of our patients and those in whom we were unable to confirm the nerve were excluded from this 
study. The overall VN identification rate was $81 \%$. Giovagnorio investigated variations of the $\mathrm{VN}$ using ultrasonography [10]. They reported that the VN ran anterior to the common carotid artery in $4.3 \%$ of 144 subjects and medial to it in $1.2 \%$. Ha also investigated the variations of $\mathrm{VN}$ anatomy, reporting that the nerve ran posterior to the common carotid artery in $13.8 \%$ and medial to the vessel in $0.3 \%$ [11]. During CEA, the surgeon cannot find a variant VN located medial or posterior to the carotid artery. Visual identification of the recurrent laryngeal nerve (RLN) during thyroid surgery is associated with lower rates of permanent RLN palsy and is considered the gold standard by many authors [12] [13] [14]. Jatzko et al. reviewed 10 reports covering 12,211 thyroid operations and found a lower rate of RLN palsy in the patients with nerve identification than in those without nerve identification $(2.7 \%$ vs $7.9 \%$ for temporary palsy and $1.2 \%$ vs $5.2 \%$ for permanent palsy) [14]. Especially in patients with visual integrity, the mechanisms of nerve injury are still not well understood [14]. We also must consider the possible side effects of continuous VNM. The same mechanism of VN stimulation was used during continuous VNM as that employed to treat drug-resistant epilepsy and depression [15] [16] [17]. Chronic VN stimulation over several months may lead to side effects, with various types of laryngopharyngeal dysfunction, including hoarseness, cough, pharyngitis, throat discomfort, laryngeal muscle spasm, and dyspnea, being detected after long-term stimulation for weeks or months [15] [16] [17]. However, no complications were seen in our patients after short-term stimulation. In this study, the mean continuous VNM time was 68 minutes, which is a very short duration of VN stimulation, but we cannot exclude the possibility that VN palsy may be induced by continuous VNM. Regarding complete EMG signal loss, the effect of VN fatigue must be considered [18] [19]. However, the lower frequency limit for fatigue of vegetative nerves is more than $10 \mathrm{~Hz}$, while we used $3 \mathrm{~Hz}$ for stimulation. In conclusion, continuous VNM was safe and it seems worthwhile to continue investigations in order to elucidate the mechanism of VN injury during CEA.

\section{Conclusion}

This is the first report of continuous VNM during CEA using new small plated form stimulation electrode. We performed postoperative laryngoscope examination for all cases. From continuous VNM and post CEA laryngoscope findings, we speculated the cause of $\mathrm{VN}$ injury was stretch effect during the caudal side ICA manipulation. Although vocal fold EMG was normal at the end point of CEA, there were six cases revealed postoperative VN palsy. These findings created a hypothesis the delayed VN injury mechanism after CEA may exist. The VN monitoring may worth trying for elucidate the mechanism of VN injury of CEA.

\section{References}

[1] Featherstone, R.L., Brown, M.M. and Coward, LJ. (2004) International Carotid Stenting Study: Protocol for a Randomised Clinical Trial Comparing Carotid Stent- 
ing with Endarterectomy in Symptomatic Carotid Artery Stenosis. Cerebrovascular Diseases, 18, 69-74. https://doi.org/10.1159/000078753

[2] Silver, F.L., Mackey, A., Clark, W,M,. Brooks, W., Timaran, C.H., Chiu, D., Goldstein, L.B., Meschia, J.F., Ferguson, R.D., Moore, W.S., Howard, G. and Brott, T.G. (2011) Safety of Stenting and Endarterectomy by Symptomatic Status in the Carotid Revascularization Endarterectomy Versus Stenting Trial (CREST). Stroke, 42, 675-680. https://doi.org/10.1161/STROKEAHA.110.610212

[3] Curran, A.J., Smyth, D., Sheehan, S.J., Joyce, W., Hayes, D.B. and Walsh, M.A. (1997) Recurrent Laryngeal Nerve Dysfunction Following Carotid Endarterectomy. $J R$ Coll Surg Edinb, 42, 168-170.

[4] Beasley, W.D. and Gibbons, C.P. (2008) Cranial Nerve Injuries and the Retrojugular Approach in Carotid Endarterectomy. Ann R Coll Surg Engl, 90, 685-688.

https://doi.org/10.1308/003588408X318138

[5] Chiang, F.Y., Lu, I.C., Kuo, W.R., Lee, K.W., Chang, N.C. and Wu, C.W. (2008) The Mechanism of Recurrent Laryngeal Nerve Injury during Thyroid Surgery-The Application of Intraoperative Neuromonitoring. Surgery, 143, 743-749.

https://doi.org/10.1016/j.surg.2008.02.006

[6] Roberts, J.W. (2008) Elucidating Mechanisms of Recurrent Laryngeal Nerve Injury during Thyroidectomy and Parathyroidectomy. Journal of the American College of Surgeons, 206, 123-130. https://doi.org/10.1016/j.jamcollsurg.2007.07.017

[7] Tomonori, T., Minoru, K., Norihiro, S., Katsuya, U., Takayuki, K. and Yoji, N. (2012) Vagus Nerve Neuromonitoring during Carotid Endarterectomy. Perspectives in Vascular Surgery and Endovascular Therapy, 24, 137-140. https://doi.org/10.1177/1531003512472239

[8] Tamaki, T., Node, Y., Saitou, N., Saigusa, H., Yamazaki, M. and Morita, A. (2013) Vernet's Syndrome after Carotid Endarterectomy. Perspectives in Vascular Surgery and Endovascular Therapy, 25, 65-68. https://doi.org/10.1177/1531003514525476

[9] Tamaki, T., Node, Y., Saitou, N. and Saigusa, H. (2013) Observation of Vocal Fold and Pharyngeal Paralysis after Carotid Endarterectomy Using a Magnifying Laryngoscope. World Journal of Surgery, 37, 911-914. https://doi.org/10.1007/s00268-013-1920-1

[10] Giovagnorio, F. and Martinoli, C. (2001) Sonography of the Cervical Vagus Nerve: Normal Appearance and Abnormal Findings. American Journal of Roentgenology, 76, 745-749. https://doi.org/10.2214/ajr.176.3.1760745

[11] Ha, E.J., Baek, J.H., Lee, J.H., Kim, J.K. and Shong, Y.K. (2011) Clinical Significance of Vagus Nerve Variation in Radiofrequency Ablation of Thyroid Nodules. European Radiology, 21, 2151-2157. https://doi.org/10.1007/s00330-011-2167-6

[12] Dionigi, G., Chiang, F.Y., Rausei, S., Wu, C.W., Boni, L., Lee, K.W., Rovera, F., Cantone, G. and Bacuzzi, A. (2010) Surgical Anatomy and Neurophysiology of the Vagus Nerve (VN) for Standardised Intraoperative Neuromonitoring (IONM) of the Inferior Laryngeal Nerve (ILN) during Thyroidectomy. Langenbeck's Archives of Surgery, 395, 893-899. https://doi.org/10.1007/s00423-010-0693-3

[13] Lorenz, K., Sekulla, C., Schelle. J., Schmeiss. B., Brauckhoff, M. and Dralle, H. (2010) What Are Normal Quantitative Parameters of Intraoperative Neuromonitoring (IONM) in Thyroid Surgery? Langenbeck's Archives of Surgery, 395, $901-$ 909. https://doi.org/10.1007/s00423-010-0691-5

[14] Jatzko, B., Vytiska-Bistorfer, E., Pawlik, A., Promberger, R., Mayerhofer, K. and Ott, J. (2014) The Impact of Thyroid Function on Intrauterine Insemination OutcomeA Retrospective Analysis. Reproductive Biology and Endocrinology [Serial Online]; 12, 28. http://www.rbej.com/content/12/1/28 
https://doi.org/10.1186/1477-7827-12-28

[15] Agnew, W.F. and McCreery, D.B. (1990) Considerations for Safety with Chronically Implanted Nerve Electrodes. Epilepsia, 31, 27-32.

https://doi.org/10.1111/j.1528-1157.1990.tb05845.x

[16] Bernards, C.M. (2004) An Unusual Cause of Airway Obstruction during General Anesthesia with a Laryngeal Mask Airway. Anesthesiology, 100, 1017-1018. https://doi.org/10.1097/00000542-200404000-00037

[17] Groves, D.A. and Brown, V.J. (2005) Vagal Nerve Stimulation: A Review of Its Applications and Potential Mechanisms That Mediate Its Clinical Effects. Neuroscience \& Biobehavioral Reviews, 29, 493-500.

https://doi.org/10.1016/j.neubiorev.2005.01.004

[18] Dralle, H., Sekulla, C., Haerting, J., Timmermann, W., Neumann, H.J., Kruse, E., Grond, S., Mühlig, H.P., Richter, C., Voss, J., Thomusch, O., Lippert, H., Gastinger, I., Brauckhoff, M. and Gimm, O. (2004) Risk Factors of Paralysis and Functional Outcome after Recurrent Laryngeal Nerve Monitoring in Thyroid Surgery. Surgery, 136, 1310-1322. https://doi.org/10.1016/j.surg.2004.07.018

[19] Ulmer, C., Friedrich, C., Kohler, A., Rieber, F., Basar, T., Deuschle, M., Thon, K.P. and Lamadé, W. (2011) Impact of Continuous Intraoperative Neuromonitoring on Autonomic Nervous System during Thyroid Surgery. Head Neck, 33, 976-984. https://doi.org/10.1002/hed.21564

\section{Scientific Research Publishing}

Submit or recommend next manuscript to SCIRP and we will provide best service for you:

Accepting pre-submission inquiries through Email, Facebook, LinkedIn, Twitter, etc. A wide selection of journals (inclusive of 9 subjects, more than 200 journals) Providing 24-hour high-quality service User-friendly online submission system Fair and swift peer-review system Efficient typesetting and proofreading procedure Display of the result of downloads and visits, as well as the number of cited articles Maximum dissemination of your research work

Submit your manuscript at: http://papersubmission.scirp.org/ Or contact ojmn@scirp.org 